\title{
Régulation de la synaptogenèse dans le muscle squelettique adulte
}

\author{
J. R. SANES $\left({ }^{1}\right)$, F. BACOU $\left({ }^{3}\right)$, J. COVAULT $\left({ }^{1}\right),\left({ }^{4}\right)$, Christine L. GATCHALIAN \\ $\left({ }^{i}\right)$, D. D. HUNTER $\left({ }^{1}\right)$, M. B. LASKOWSKI $\left({ }^{1}\right),\left({ }^{5}\right)$, J. P. MERLIE $\left({ }^{2}\right)$, L. J. \\ SCOTT \\ (1) Department of Anatomy and Neurobiology and $\left({ }^{2}\right)$ Department of Pharmacology \\ Washington University School of Medicine \\ 660 s. Euclid, St. Louis, MO 63110, USA. \\ $\left.{ }^{3}{ }^{3}\right)$ Département de Physiologie animale, I.N.R.A.-E.N.S.A., \\ Place Viala, 34060 Montpellier Cedex. France. \\ $\left({ }^{4}\right)$ Nouvelle adresse : Department of Physiology \\ University of Connecticut, Storrs, CT 06268, USA. \\ $\left({ }^{5}\right)$ Nouvelle adresse : Department of Physiology. St. Louis University Medical School, \\ 1402 s. Grand BIvd. St. Louis, MO 63104, USA.
}

Summary. Regulation of synaptogenesis in adult skeletal muscle.

Several behaviors that axons exhibit during reinnervation of adult skeletal muscle demonstrate that they are guided by cues that the muscle provides. For example, axons form synapses on denervated but not on normally innervated muscle fibers; axons selectively reinnervate original synaptic sites on denervated muscle fibers; regenerating axons become specialized for synaptic transmission only in regions where they contact muscle fibers; and motor axons prefer to reinnervate muscles derived from matching levels of the body's rostro-caudal axis. This chapter describes these phenomena and summarizes progress toward identifying the soluble, membrane-bound, and extracellular matrix molecules that underly them.

\section{Introduction.}

Au cours de leur croissance, les axones utilisent plusieurs voies extrinsèques pour déterminer où et quand établir des synapses. Les neurobiologistes du développement ont à répondre aux questions fondamentales : quelles sont ces voies? où sont-elles situées? comment leur expression est-elle modulée? Pour y parvenir, nous avons choisi d'étudier la jonction neuromusculaire des vertébrés. En effet, la jonction neuromusculaire est peut-être la plus simple et certainement la mieux connue de toutes les synapses, quantité d'informations étant disponibles quant à leur structure, leur fonction et leur développement. De plus, la spontanéité de la réinnervation musculaire après lésion permet d'étudier la formation synaptique sur un système postembryonnaire pratique. Cet exposé rend compte de nos travaux effectués sur les facteurs guidant les axones lors de la réinnervation 
des muscles squelettiques, et les situe dans le contexte du grand nombre d'études réalisées par d'autres groupes sur ce sujet.

\section{Réinnervation précise aux sites synaptiques primitifs.}

Les fibres musculaires énervées évoluent selon plusieurs axes : elles s'atrophient, acquièrent de nouvelles propriétés métaboliques, électriques et contractiles, et les récepteurs à l'acétylcholine (AChRs) se répandent sur toute leur surface. Néanmoins, les sites synaptiques primitifs conservent nombre de leurs propriétés spécifiques et demeurent identifiables plusieurs mois après la dégénérescence des terminaisons nerveuses. Ainsi, les AChRs demeurent concentrés au niveau de la membrane synaptique à une densité voisine de $10^{4} / \mu \mathrm{m}^{2}$, alors même que de nouveaux récepteurs sont insérés sur la membrane extrasynaptique à une densité de $10^{2}-10^{3} / \mu \mathrm{m}^{2}$ (Salpeter and Loring, 1985). De même, les digitations membranaires qui s'invaginent dans la membrane postsynaptique et les amas de noyaux associés avec la plaque motrice persistent. Tello (1907) fut le premier à rapporter que les axones en régénération formaient de nouvelles jonctions neuromusculaires de préférence sur ces sites synaptiques primitifs. Cette découverte a été confirmée depuis sur les muscles d'amphibiens, d'oiseaux et de mammifères réinnervés soit par leur propre nerf, soit par un nerf différent (Gutmann and Young, 1944 ; Aitken, 1950; Landmesser, 1972 ; Bennett and Pettigrew, 1976 ; Letinsky et al., 1976). Bien que les axones puissent parfois former des synapses « ectopiques " sur des zones initialement extrasynaptiques des fibres musculaires (fig. 1 ; voir ci-dessous), la réinnervation précise aux sites primitifs est généralement la règle, et la spécificité topographique de ce processus est remarquable. Ainsi, dans les muscles de grenouille réinnervés après écrasement de leur nerf, plus de $90 \%$ des sites synaptiques primitifs sont réinnervés et plus de $95 \%$ des contacts nerf-muscle formés se situent aux sites originels, bien que ces sites occupent moins de $0,1 \%$ de la surface de la fibre musculaire (Sanes et al., 1978). Ces résultats attestent donc l'existence de facteurs concentrés aux sites synaptiques primitifs, susceptibles d'être reconnus par les axones en régénération.

Certains de ces facteurs sont associés avec la lame basale (LB) qui s'intercale dans la fente synaptique à la jonction neuromusculaire. Les expériences amenant à cette conclusion sont résumées sur la figure 2. Les muscles sont énervés, lésés mécaniquement de façon à provoquer la dégénérescence des fibres, et traités aux rayons $X$ pour empêcher la régénération de nouveaux tissus musculaires. Les enveloppes de LB des fibres musculaires survivent à ces traitements, et les sites synaptiques primitifs peuvent être identifiés sur la LB même après dégénérescence de tous les éléments cellulaires. Les axones régénérant vers la région lésée contactent les gaines de lame basale, et plus de $95 \%$ des contacts détectés par microscopie électronique sont situés avec précision aux sites synaptiques originels. Ainsi, la réinnervation est topographiquement aussi sélective en l'absence de cellule musculaire qu'en leur présence (Sanes et al., 1978). De plus, les parties de l'axone qui contactent la LB synaptique se différencient en 

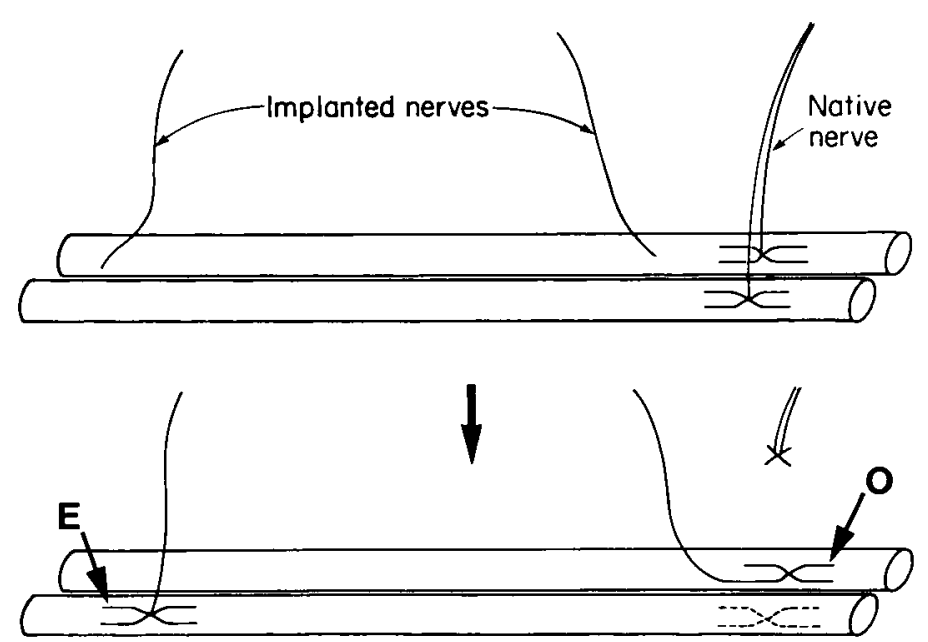

FIG. 1. - Les axones réinnervent de préférence les sites synaptiques origine/s (O) dans les muscles énervés, mais peuvent former dans certains cas des synapses ectopiques (E). Dans les muscles innervés, les axones implantés forment peu ou pas de synapses (d'après Sanes and Covault, 1985).

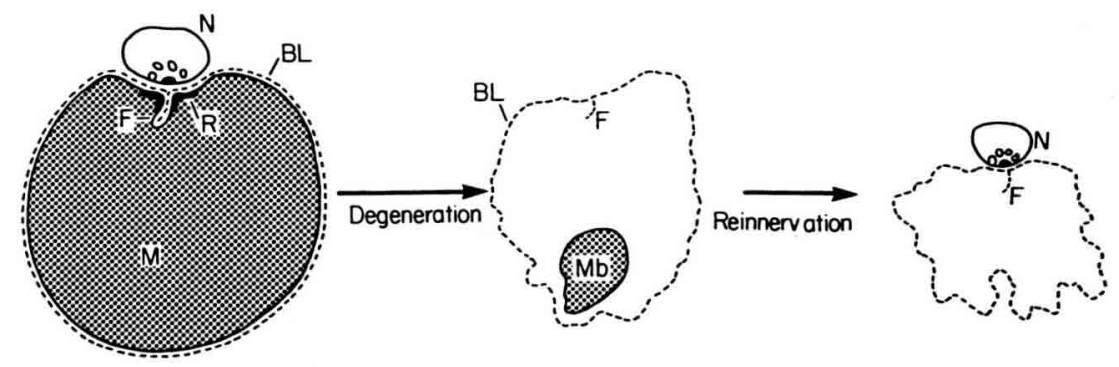

FIG. 2. - Schéma de l'expérience démontrant un rôle de la lame basale synaptique dans la régénération des synapses. $\mathrm{N}$, terminaison nerveuse : $\mathrm{M}$, fibre musculaire; $\mathrm{F}$, digitation jonctionnelle; $\mathrm{R}$, récepteur de l'acétylcholine; $\mathrm{BL}$, lame basale; $\mathrm{Mb}$, cellule satellite myoblastique. Voir le texte pour explications (d'après Sanes and Chiu, 1983).

terminaisons nerveuses, selon plusieurs critères: morphologiques, électriques et immunohistochimiques (Sanes et al., 1978 ; Glicksman and Sanes, 1983). Ainsi, il existe des constituants de la LB synaptique reconnus par les axones en régénération, avec lesquels ils réagissent.

Les bases moléculaires de cette spécialisation fonctionnelle ont été identifiéespar voies histochimiques: de nombreux antisera et anticorps monoclonaux marquent la LB synaptique beaucoup plus intensément que la LB extrasynapti- 
que, définissant ainsi des antigènes «synaptiques" (Sanes and Hall, 1979; Anderson and Fambrough, 1983 ; Sanes and Chiu, 1983 ; Fallon et al., 1985). L'héparane sulfate protéoglycane est l'un de ces antigènes (Anderson and Fambrough, 1983) ; un autre est semblable sinon identique à la protéine " agrine » qui, selon McMahan et ses collaborateurs, provoque l'aggrégation des AChRs sur la membrane des myotubes (Fallon et al., 1985). Nous avons identifié une protéine de $185 \mathrm{kd}$ comme étant l'antigène reconnu par un ensemble de quatre anticorps monoclonaux qui tous marquent sélectivement la LB synaptique (Hunter et al.,1987; fig. 3). Enfin, plusieurs anticorps monoclonaux se lient à la LB extrasynaptique et non à la LB synaptique, définissant ainsi des épitopes inexistants dans la fente synaptique (Sanes, 1982; Sanes and Chiu, 1983; Gatchalian et al., 1985). Il n'a pas encore été démontré que ces molécules concentrées (ou absentes de) à la LB synaptique représentaient un signal susceptible d'être reconnu par les axones. Cependant, des travaux en ce sens sont en cours, et des tests biologiques tels que ceux que nous décrirons plus loin permettent de déterminer le rôle des antigènes reconnus par les anticorps déjà en notre possession.
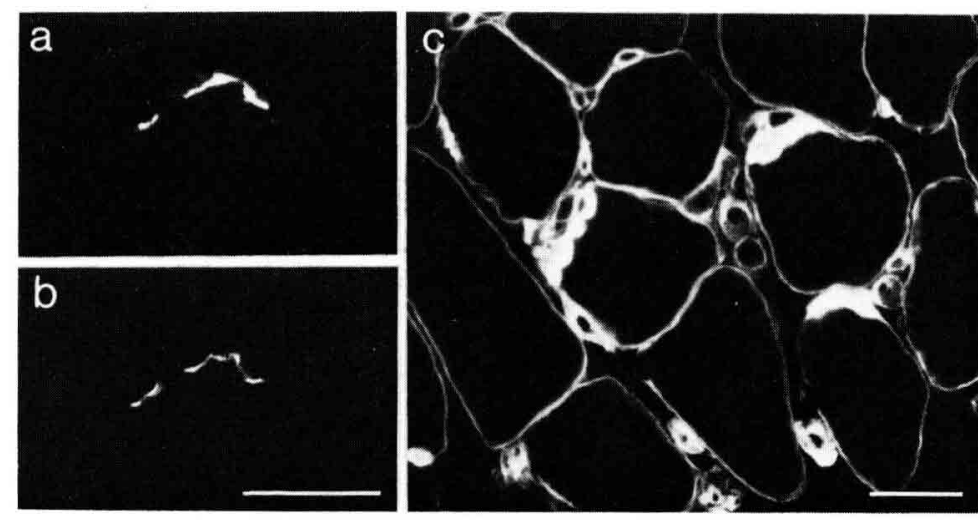

FIG. 3. - Des anticorps monoc/onaux contre certains constituants de la LB sont concentrés aux sites synaptiques (a) (b correspond au même champs marqué à l' $\alpha$-bungarotoxine rhodaminée qui se lie aux AChRs, marquant ainsi les sites synaptiques) ou bien sont distribués sur l'ensemb/e de la $\angle B$ des fibres musculaires (c). a-c sont des sections au cryostat de muscles de rats adultes marquées par immunofluorescence; $d$ montre un Western blot d'un extrait de LB réagissant avec l'anticorps marquant en a. II apparaît une bande immunoréactive majeure voisine de $185 \mathrm{Kd}$. Echelle a-c $=20 \mu \mathrm{m}$.

Parallèlement aux études immunologiques, nous avons aussi utilisé des lectines afin d'analyser les glycogonjugués de la LB synaptique (Sanes and Cheney, 1982). Un grand nombre de lectines sont connues pour leur liaison hautement spécifique avec une fraction mono- ou oligosaccharidique caractéristique ; conjuguées à un fluorophore, elles représentent de remarquables sondes histochimiques de la structure des glucides. De nombreuses lectines marquent à 
la fois la LB synaptique et extrasynaptique, mais au moins deux se lient spécifiquement à la LB synaptique. Ces deux lectines, l'aglutinine de Dolichos biflorus (DBA) et l'isoaglutinine B4 de Vicia villosa (VVA) sont spécifiques des résidus terminaux $\mathrm{N}$-acétylgalactosamine (GalNAc), démontrant donc que le glucide synaptique se termine en un GalNAc ; comme l'ont montré des analyses complémentaires, celui-ci est dans une configuration $\beta$-anomérique, relativement inhabituelle. En utilisant ces lectines en chromatographie d'affinité, nous avons trouvé que la forme à queue collagénique (asymétrique) de l'acétylcholinestérase - que l'on savait déjà être concentrée à la LB synaptique - est une molécule qui porte des résidus terminaux $\beta$ GalNAc. Fait intéressant, d'autres molécules synaptiques bien moins caractérisées, dont un glycolipide de la membrane postsynaptique, portent aussi ce glucide synaptique. Ces résultats montrant qu'au moins deux (et probablement davantage) molécules spécifiques de la synapse comportent un glucide synaptique suggèrent que ce dernier joue un rôle dans la formation ou la fonction de la synapse. Des données apportées par d'autres systèmes suggèrent que les glucides pourraient servir de marqueurs de reconnaissance synaptogénique, possibilité qui pourrait être testée à la jonction neuromusculaire.

\section{Aptitude et résistance à la formation des synapses.}

Si un nerf moteur est coupé et son extrémité distale suturée dans un muscle squelettique, ses axones survivent et se ramifient localement mais ne forment pas ou peu de synapses sur les fibres musculaires innervées qu'ils rencontrent. Si par la suite, le nerf propre du muscle est sectionné, les axones croissent bien davantage et forment rapidement des synapses. Ces observations, rapportées pour la première fois par Elsberg (1917) et plusieurs fois confirmées depuis (voir Aitken, 1950 ; Jansen et al., 1973; Weinberg et al., 1981), montrent que les muscles sont capables de contrôler leur aptitude à la formation synaptique selon l'état de leur innervation. Bien que, comme nous l'avons vu précédemment, le nerf implanté forme préférentiellement des plaques motrices aux sites synaptiques primitifs, les axones peuvent former, dans les muscles de mammifères au moins, des synapses nouvelles ou "ectopiques" s'ils sont implantés loin des sites synaptiques ou si ceux-ci leur sont rendus inaccessibles. Ce phénomène de la synaptogenèse " ectopique » indique que la résistance à la formation synaptique des fibres musculaires innervées n'est pas due seulement à l'occupation physique d'un seul site compétent, mais correspond plutôt à une propriété s'étendant sur toute la longueur de la fibre musculaire.

Comment la fibre musculaire informe-t-elle le nerf de sa prédisposition à l'innervation ? II paraît vraisemblable que les muscles innervés ou énervés (ou des muscles actifs ou inactifs) produisent en quantités différentes certain(s) facteur(s) influençant les axones. Dans cet ordre d'idée, les AChRs sont concentrés au niveau de la membrane postsynaptique, et les ARNms de leurs sous-unités sont localisés dans les zones synaptiques (Merlie and Sanes, 1985). Après énervation, le niveau des ARNms des AChRs augmente (Merlie et al., 1984 ; Goldman et al., 
1985 ; Klarsfeld and Changeux, 1985 ; Covault et al., 1986), et de nouveaux AChRs sont synthétisés et insérés sur toute la surface de la fibre musculaire. Le contrôle neural de la synthèse des AChRs s'effectue en grande partie par l'intermédiaire de l'activité musculaire, puisqu'on observe des AChRs extrasynaptiques sur des muscles innervés mais paralysés, et non sur des muscles énervés stimulés directement. De même, les axones implantés peuvent former des synapses " ectopiques " sur des muscles innervés et paralysés, mais non sur des muscles énervés et stimulés (Jansen et al., 1973). Nous avons proposé que les gènes des sous-unités des AChRs constituent les membres d'une "famille de gènes spécifiques de la synapse » transcrits de préférence par les noyaux associés à la plaque motrice des muscles actifs, et dans les noyaux non synaptiques lorsque les muscles deviennent inactifs (Merlie and Sanes, 1985, 1986). Il est tentant de penser que les gènes qui codent pour les facteurs neuroactifs contrôlant l'aptitude musculaire à l'innervation soient membres de cette famille, et que leur expression soit modulée parallèlement à celle des AChRs.

Un tel facteur pourrait être un facteur de croissance soluble provoquant la régénération des axones moteurs. L'hypothèse de l'existence d'un tel facteur a été faite il y a quelques années, à partir d'études menées sur le «Nerve Growth Factor " (NGF) que les tissus cibles sensitifs et sympathiques utilisent pour contrôler leur innervation. En fait, plusieurs groupes ont montré que des extraits de muscles, ou des milieux qu'ils ont conditionnés, peuvent augmenter la survie et la différenciation des motoneurones en culture, ces extraits étant plus actifs lorsqu'ils sont préparés à partir de muscles en développement ou énervés (Henderson et al., 1983; Slack et al., 1983 ; Nurcombe et al., 1984). Parallèlement, des anticorps préparés contre un de ces facteurs peuvent bloquer partiellement le bourgeonnement axonal lorsqu'ils sont injectés dans des muscles adultes paralysés (Gurney et al., 1986). Ainsi, la synthèse accrue et/ou la sécrétion d'un "Motor Nerve Growth Factor" par les muscles énervés ou paralysés pourraient stimuler la croissance axonale et de ce fait favoriser la réinnervation.

Plusieurs observations faites sur le contrôle de la formation des synapses " ectopiques» (Sanes and Covault, 1985), et la nécessité d'interactions avec la surface cellulaire lors de la synaptogenèse, suggèrent que les muscles utilisent des molécules situées à la surface de la cellule aussi bien que des facteurs solubles pour moduler leur aptitude à l'innervation. La "Neural Cell Adhesion Molecule " (NCAM) représente une postulante majeure pour ce rôle. Deux séries de données étayent cette suggestion. Premièrement, les niveaux de la NCAM in vivo sont modulés en parallèle avec la prédisposition des fibres musculaires à l'innervation (Rieger et al., 1985 ; Covault and Sanes, 1985-1986 ; Covault et al., 1986 ; Moore and Walsh, 1986 ; Cashman et al., 1987). Les myotubes embryonnaires sur lesquels des synapses se forment facilement sont riches en NCAM; la NCAM disparait au cours de la période périnatale, et est pratiquement absente des zones extrasynaptiques des fibres musculaires adultes, qui sont réfractaires à l'hyperinnervation : cependant, la NCAM demeure concentrée (à et) près de la membrane postsynaptique du muscle adulte. Après énervation, le muscle étant à nouveau réceptif à l'innervation, la NCAM réapparaît sur toute la fibre musculaire, une 
induction qui est accompagnée et est certainement due à l'augmentation des ARNms de la NCAM (fig. 4); de même la paralysie induit une accumulation de NCAM. Dans les muscles énervés ou paralysés, la NCAM apparaît non seulement dans les fibres musculaires mais aussi dans des zones interstitielles situées entre les fibres, point que nous discuterons plus loin. De plus, la NCAM est abondante sur le cône de croissance des axones moteurs en régénération, qui sont ainsi capables via un mécanisme de liaison homophile, de réagir à la NCAM musculaire. Enfin, la NCAM disparaît des zones extrasynaptiques des fibres musculaires (et des zones interstitielles) lorsque le muscle est réinnervé et perd sa capacité à former des synapes.
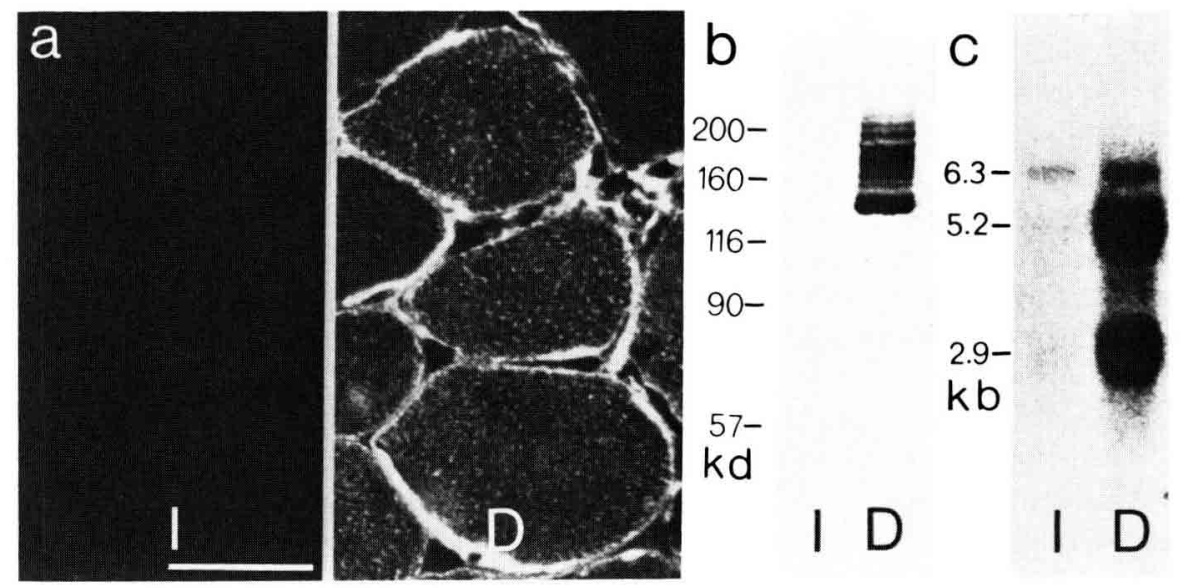

FIG. 4. - Après énervation, les quantités de NCAM et de leur ARN augmentent dans les muscles squelettiques: par immunoflorescence (a), Western blot (b) et Northern blot (c). «I " et «D » correspondent respectivement aux muscles innervés et énervés (b et c, d'après Covault et al., 1986). Echelle en a $=50 \mu \mathrm{m}$.

Le deuxième point étayant le rôle médiateur de la NCAM dans les interactions nerf-muscle découle d'expériences utilisant des anticorps. Bien qu'aucune de ces études n'ait été effectuée in vivo, deux équipes ont montré que de l'anti-NCAM pouvait perturber les interactions nerf-muscle in vitro. Un point de désaccord existe entre ces deux groupes, l'un (Grumet et al., 1982 ; Rutishauser et al., 1983) trouvant des effets avec I'anti-NCAM seul, l'autre (Bixby and Reichardt, 1987 ; Bixby et al., 1987) rapportant que l'anti-NCAM n'est efficace que combiné avec des anticorps contre d'autres systèmes d'adhérence. Ce dernier résultat suggère que la NCAM n'est seulement qu'un des systèmes d'adhérence participant aux interactions nerf-muscle, conclusion en accord avec les études sur la LB précédemment décrites, et par le fait qu'un complexe d'adhérence lamininehéparane sulfate protéoglycane situé à la surface des fibres musculaires innervées augmente après énervation (Chiu et al., 1986). Quoiqu'il en soit, il n'en 
demeure pas moins une hypothèse à tester de façon plus approfondie sur le rôle de la NCAM, une des molécules susceptible d'être utilisée par les muscles pour informer les nerfs sur leur aptitude à l'innervation.

\section{Comment les axones retrouvent-ils les sites synaptiques primitifs ?}

L'ensemble des résultats exposés dans les paragraphes précédents soulève une nouvelle question: si les axones sont capables de former des synapses " ectopiques », comment retrouvent-ils le minuscule site synaptique primitif qu'ils réinnervent préférentiellement? Le problème n'est pas simple puisque ces sites occupent seulement $0,1 \%$ de la surface de la fibre musculaire, et si ce processus de recherche s'effectuait au hasard, les filopodes des axones moteurs en régénération ne rencontreraient pas fréquemment ces sites. En fait, il y a au moins trois mécanismes potentiels susceptibles d'intervenir pour accroître la spécificité topographique de la réinnervation, en relation avec les constituants de la LB synaptique.

Un premier type de guidage est dû aux gaines de tissu conjonctif des nerfs intramusculaires. Alors que les parties distales de l'axone moteur dégénèrent après axotomie, les cellules de soutien et le tissu conjonctif des nerfs survivent. Les axones en régénération retournent généralement à la surface des fibres musculaires à l'intérieur de ces troncs nerveux, et sont de ce fait conduits aux régions synaptiques primitives (voir Gutmann and Young, 1944). Les axones peuvent traverser un certain espace pour pénétrer dans l'extrémité sectionnée d'un segment nerveux, ce qui suggère que des cellules situées à l'intérieur du bout distal du nerf libèrent un facteur soluble attirant les axones (Ramon y Cajal, 1928 ; Kuffler, 1986). Une fois dans le nerf, les axones en régénération croissent, et adhèrent certainement à la LB des tubes endoneuraux (cellule de Schwann, voir Ide et al, 1983; Scherer et Easter, 1984). Les études effectuées in vitro sur l'extension axonale sur des substrats de type LB suggèrent que le constituant actif de la LB est un complexe laminine-héparane sulfate protéoglycane (Davis et al., 1985 ; Lander et al., 1985 ; Chiu et al., 1986) reconnu par un récepteur cellulaire de la membrane neuritique (Bozyczko et Horwitz, 1986) nommé "Integrine » (Hynes, 1987). Ainsi, les troncs nerveux contiennent à la fois des molécules solubles et des molécules de la matrice extracellulaire susceptibles d'orienter la croissance axonale.

Une deuxième voie de guidage axonal découle des études immunohistochimiques faites sur des muscles énervés. Quatre molécules d'adhérence s'accumulent dans les zones interstitielles proches des sites synaptiques au cours des premiers jours suivant l'énervation : ce sont les J1, NCAM, fibronectine et un héparane sulfate protéoglycane (Sanes et al., 1986). Les dépôts interstitiels représentent seulement une fraction minime du contenu intramusculaire des trois dernières molécules : comme nous l'avons déjà signalé, fibronectine et héparane sulfate protéoglycanes sont des constituants normaux de la LB, à la fois dans les muscles innervés et énervés, alors que la NCAM n'apparaît sur l'ensemble de la surface des fibres musculaires qu'après énervation. Néanmoins, l'immunofluo- 
rescence montre clairement que ces molécules s'accumulent à l'extérieur des fibres musculaires énervées et que les dépôts interstitiels sont concentrés près des synapses. La situation est plus complexe en ce qui concerne J1. La J1 a été identifiée chez les murins comme une molécule intervenant dans l'adhérence des neurones centraux avec les astrocytes (Kruse et al., 1985), et elle est vraisemblablement très proche sinon identique à la « cytotactine » (Grumet et al., 1985) et à la " ténascine» (Chiquet-Ehrismann et al., 1986), deux molécules trouvées chez les oiseaux. La J1 est pratiquement indétectable dans les muscles normaux, tandis que dans les muscles énervés, elle est concentrée aux zones interstitielles périsynaptiques dont elle peut représenter un excellent marqueur. Puisque les axones en régénération traversent ces zones en approchant des sites synaptiques primitifs, il paraît vraisemblable qu'ils rencontrent des dépôts de macromolécules d'adhérence et que leur croissance puisse être ainsi guidée.

Des études récentes sur l'origine des dépôts interstitiels de molécules d'adhérence laissent penser que leur synthèse s'effectue, au moins en partie, au niveau d'une population de fibroblastes proliférant après énervation dans les zones périsynaptiques. Les fibroblastes sont peu abondants dans les muscles, et on sait depuis peu de temps que l'énervation stimule leurs divisions (Murray and Robbins, 1982). Récemment, Connor and McMahan (1987) ont montré que ces divisions - et subséquemment le nombre de fibroblastes - sont plus nombreuses près plutôt que loin des plaques motrices, résultats que nous avons confirmés sur le muscle de rat (fig. 5 ; Gatchalian and Sanes, 1987). L'immunohistochimie en microscopie électronique révèle que la NCAM interstitielle est située à la surface de ces cellules, tandis que la J1 est associée avec les fibrilles de collagène contiguës aux processus fibroblastiques. En utilisant des méthodes d'immunofluorescence et d'immunoprécipitation, nous avons trouvé que des fibroblastes cultivés, issus de muscles énervés, synthétisent les NCAM, J1, fibronectine et héparane sulfate protéoglycanes (fig. 6 ; Gatchalian and Sanes, 1987). On ne sait pas encore si ces fibroblastes correspondent à un seul type cellulaire, ou si ce sont des fibroblastes classiques réagissant à une information localisée, dépendante du nerf. Dans l'un ou l'autre cas cependant, le fait que les fibroblastes puissent exprimer de multiples molécules d'adhérence suggère un nouveau type de voies susceptibles de guider les axones.

Une voie supplémentaire dans le guidage axonal découle des études faites sur la distribution spatiale des synapses «ectopiques» formées au cours de la réinnervation du muscle de rat. Dans le soléaire, les axones implantés forment de nombreuses synapses « ectopiques » dans les parties distales du muscle, mais peu de synapses dans une région de approximativement $1 \mathrm{~mm}$ située autour de la zone centrale des plaques motrices originelles (Frank et al., 1975). Un comportement semblable a été rapporté sur le diaphragme après énervation néonatale (Bennett and Pettigrew, 1974). II est possible que ces schémas découlent de mécanismes déjà discutés: les axones qui croissent suffisamment près du site synaptique primitif pourraient rencontrer des dépôts interstitiels de macromolécules d'adhérence, des fibroblastes, des branches nerveuses, et soient ainsi stimulés pour croître au-delà de la zone périsynaptique jusqu'aux sites synaptiques. Une alternative cependant — proposée d'ailleurs par Bennett et Pettigrew 

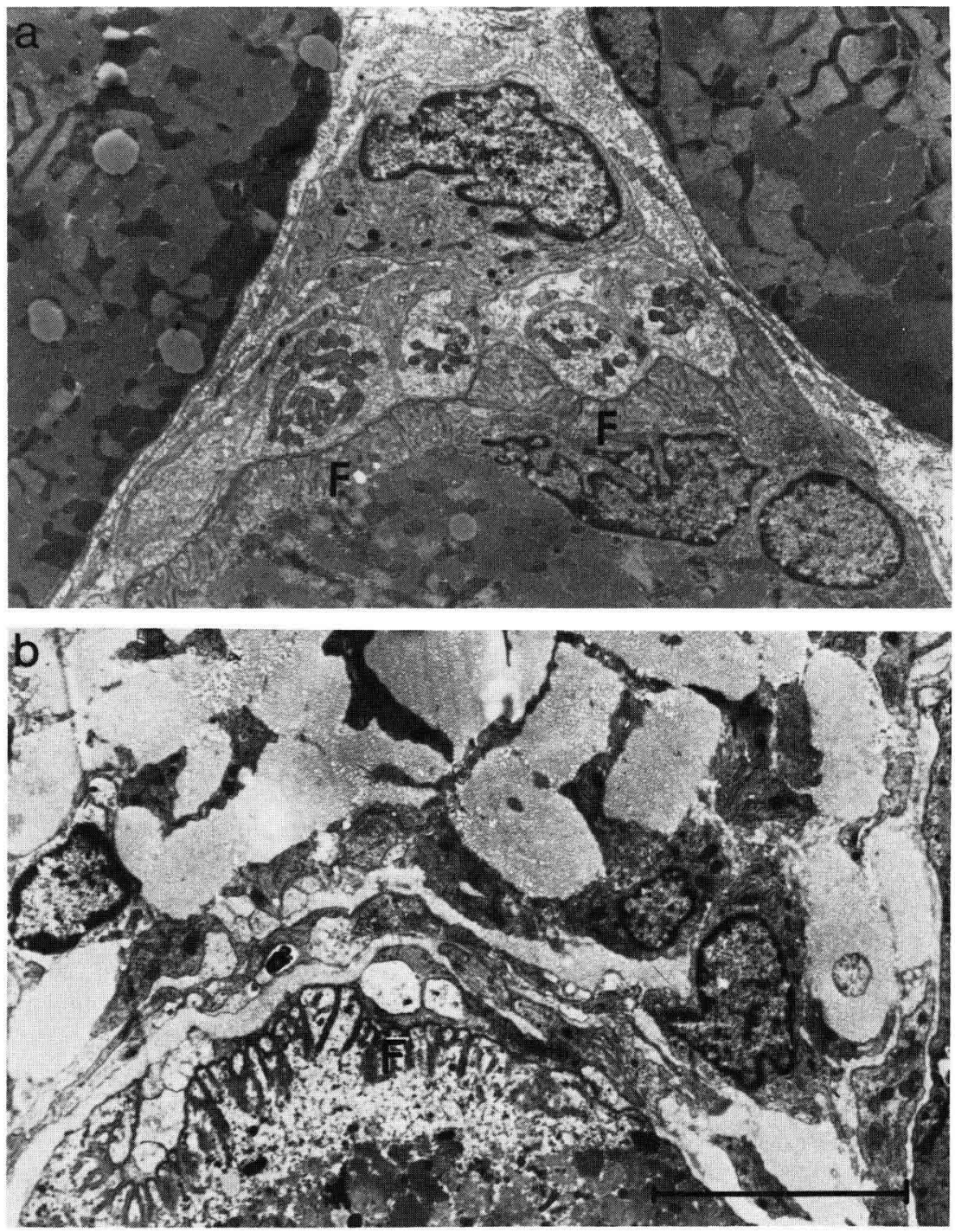

FIG. 5. - Micrographie électronique de sites synaptiques de diaphragme innervé (a) et énervé (b) de rat montrant la prolifération des fibroblastes qui intervient près des plaques motrices énervées. $\mathrm{F}$, digitations jonctionnelles. Echelle $=5 \mu \mathrm{m}$.

(1976) - est que l'aptitude des fibres musculaires à former des synapses est un phénomène graduel, les zones périsynaptiques étant plus réfractaires à l'innervation que ne le sont les zones distales. A la lumière de récentes études in vitro montrant que l'accumulation des AChRs à la jonction nerf-muscle est accompagnée par leur diminution dans les zones adjacentes (Anderson et al., 1977; Kuromi and Kidikoro, 1984), il n'est pas impensable que d'autres molécules importantes dans la formation synaptique soient peu abondantes dans les zones périsynaptiques. 

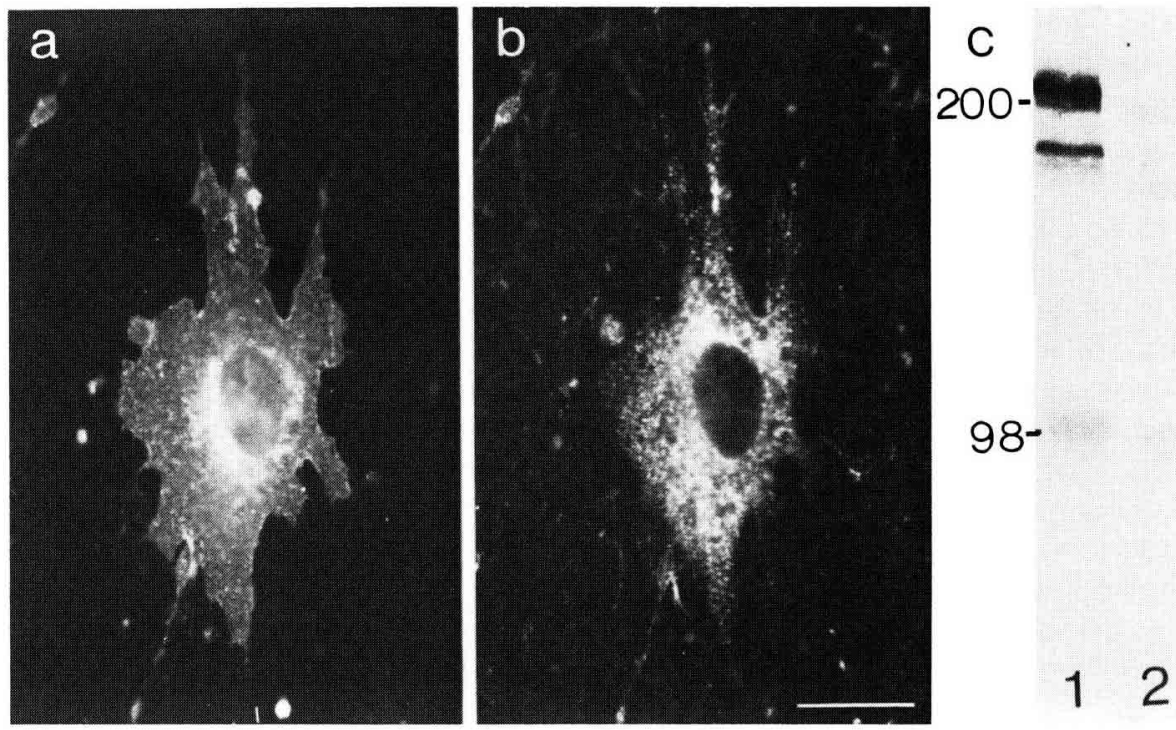

FIG. 6. - a, b. Fibroblastes cultivés issus de muscle squelettique énervé, double marquage effectué par l'anti-NCAM (a) et la fibronectine (b). Echelle $=25 \mu \mathrm{m}$. c. Immunoprécité de J1 d'une culture de fibroblastes, marquée à la méthionine $-S^{35}$, de muscle énervé. Les lignes 1 et 2 montrent respectivement les sera immuns et preimmuns.

\section{Réinnervation sélective de position.}

Les voies précédemment décrites sont «spécifiques» en ce sens qu'elles limitent la formation des synapses à un état particulier du muscle (énervé) ou à des zones déterminées de la fibre musculaire (sites synaptiques primitifs). Cependant, la marque de la spécificité neurale est considérée généralement comme étant la discrimination entre des cellules cibles potentielles, la synaptogenèse s'effectuant entre des partenaires particulièrement appropriés. Selon cette définition classique, la réinnervation telle que nous l'avons décrite n'est pas particulièrement sélective : un axone moteur "étranger » et même un axone préganglionnaire autonome peuvent innerver des muscles énervés mais non pas des muscles innervés, formant de préférence des synapses aux sites synaptiques primitifs (Landmesser, 1972 ; Jansen et al., 1973 ; Bennett and Pettigrew, 1976). Chez les vertébrés inférieurs, une réinnervation sélective des muscles et des types de fibres musculaires par leurs propres axones a été montrée par électrophysiologie (Elizalde et al., 1983; Wigston, 1986). Cependant, la plupart des tentatives effectuées pour démontrer la réinnervation sélective des muscles de mammifères adultes a été infructueuse : les nerfs moteurs natifs et étrangers paraissent avoir des potentialités de réinnervation musculaire comparables, lorsqu'on considère l'accès et le nombre relatif des axones (Bernstein and Guth, 1961 ; voir références complémentaires dans Wigston and Sanes, 1985). Ces résultats décourageants ont été utilisés pour proposer que les facteurs (hypothétiques) intervenant pour 
guider l'innervation spécifique chez l'embryon sont peu exprimés chez l'adulte, et que par conséquent les muscles adultes sont de piètres systèmes pour étudier ce type de sélectivité.

En reprenant cette conclusion, nous avons émis l'hypothèse qu'axones et muscles pourraient s'apparier sur la base de leur position dans l'axe rostro-caudal. Chez les animaux normaux, les muscles, issus des somites disposés segmentairement, sont innervés généralement par les neurones des niveaux correspondants de la moelle épinière. Tandis qu'un tel arrangement chez l'embryon est pour l'essentiel le résultat des relations spatiales existant entre les axones en croissance et les muscles, il nous a semblé que le niveau segmentaire pouvait être un paramètre intéressant permettant d'étudier une réinnervation sélective. Nous avons donc étudié la réinnervation des muscles intercostaux, chacun d'entre eux se développant à partir d'un seul somite et étant normalement innervé par les motoneurones situés dans le segment correspondant de la moelle épinière. Des fragments d'intercostal d'un des différents niveaux ont été transplantés en un site commun, au niveau du cou du rat, et le tronc sympathique cervical a été implanté dans le muscle. Ce nerf a été choisi car ses axones proviennent de différents niveaux de la moelle épinière, peuvent innerver les muscles squelettiques, et comme l'ont montré Purves et al. (1981), sont capables d'innerver sélectivement les neurones sympathiques. Après réinnervation, nous avons fait un enregistrement intracellulaire des fibres musculaires du greffon et stimulé individuellement les racines ventrales pour déterminer à quels segments correspondaient les axones innervant les muscles. Les résultats montrent que l'origine segmentaire de l'innervation reçue par les muscles diffère: les muscles d'un niveau rostral reçoivent une quantité disproportionnée de leur innervation depuis les axones rostraux du nerf, tandis que les muscles caudaux sont innervés par un ensemble d'axone d'origine caudale (Wigston and Sanes, 1982, 1985). Ces résultats montrent que les axones peuvent faire la distinction parmi les muscles squelettiques adultes, selon un schéma correspondant à leur position d'origine dans l'axe rostro-caudal.

Nous avons développé cette analyse en étudiant l'innervation et la réinnervation des muscles squelettiques par les axones moteurs. Nous avons choisi pour cela des muscles innervés par des axones issus de plusieurs segments spinaux mais qui pénètrent dans le muscle en un seul nerf : le Serratus anterior en est un exemple (fig. 7a). Par des méthodes complémentaires électrophysiologiques et histologiques, nous avons trouvé que l'axe rostro-caudal du pool moteur spinal est systématiquement organisé en un axe rostro-caudal à la surface des muscles (fig. 7b; Laskowski and Sanes, 1987a). De plus, après lésion nerveuse, la réinnervation s'effectue selon une disposition sélective : les axones rostraux et caudaux réinnervent de préférence respectivement les secteurs rostraux et caudaux du muscle (fig. 7c; Laskowski and Sanes, 1987b). Des résultats semblables sur la topographie de l'innervation normale et sur la sélectivité de la réinnervation ont été obtenus sur un deuxième muscle, le diaphragme (Laskowski and Sanes, 1987a, b). Ainsi, lorsque la position rostro-caudale est le paramètre étudié, la réinnervation des muscles des mammifères adultes est indiscutablement sélective. 


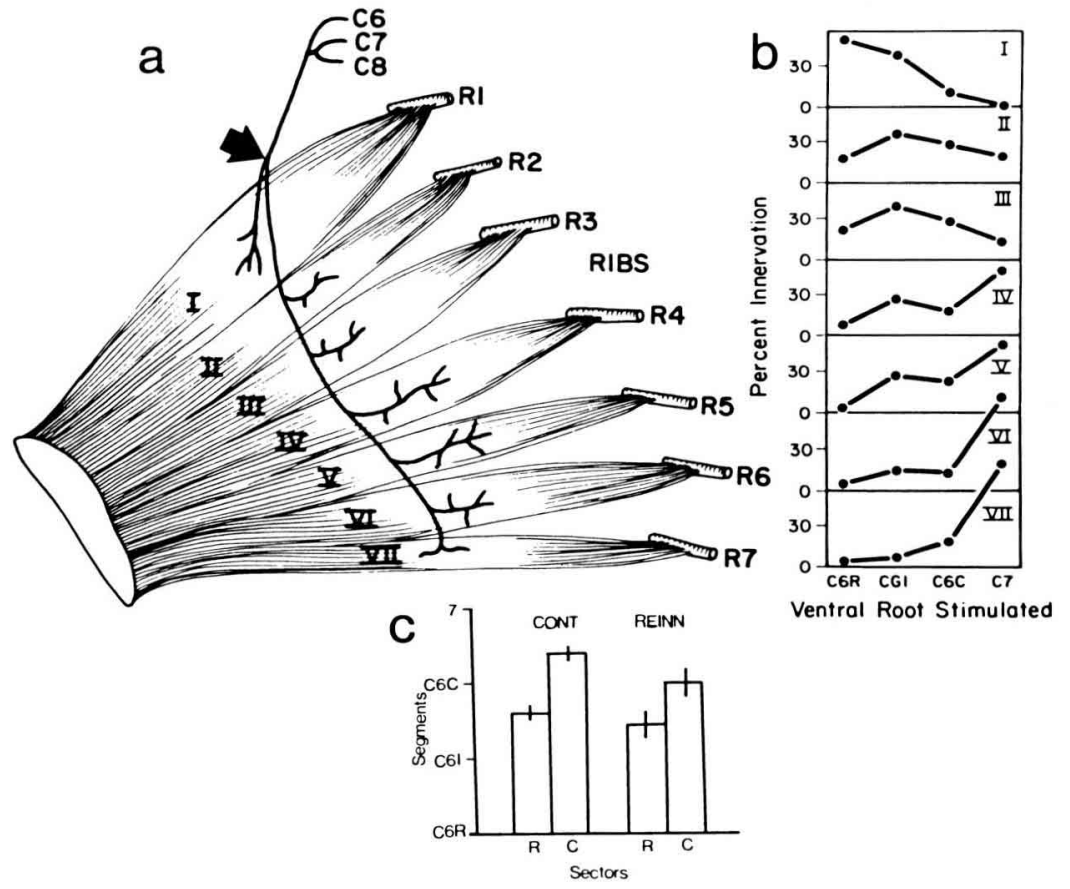

FIG. 7. - a. Diagramme du musc/e Serratus anterior, innervé par un seul nerf issu des niveaux C6-8 de la moelle épinière. b. Origine segmentaire des réponses synaptiques de chaque secteur du Serratus anterior, mise en évidence par enregistrement intracellulaire ( $R$, I et $C$ représentent respectivement les radicelles rostrales, intermédiaires et caudales de la racine C6). c. Origine movenne des réponses des secteurs rostraux (R:II-IV) et caudaux (C:V-III) du Serratus anterior normal et réinnervé démontrant significativement $(\mathrm{p}<0,01)$ une réinnervation sélective positionne/le. Le secteur I est exclu de l'analyse, car il n'est pas complètement énervé chez certains animaux ( $a$ et $\mathbf{b}$, d'après Laskowski and Sanes, 1987).

Ces études électrophysiologiques représentent une base d'étude de la formation sélective des synapses sur les muscles adultes, et fournissent en outre trois indices sur la nature de la sélectivité. Premièrement, il semble exister un système d'adéquation des axones et de leurs cibles à un niveau donné de l'axe rostro-caudal. Deuxièmement, ces voies de position peuvent ne pas être limitées à un seul système : elles peuvent être détectées soit par les axones autonomes, soit pas les axones moteurs réinnervant les muscles squelettiques, comme par les axones autonomes réinnervant les ganglions sympathiques (Purves et al., 1981). Enfin, la connectivité n'est pas étroitement spécifique, puisque les axones de pratiquement toute la moelle épinière sont capables d'innerver des muscles (ou des ganglions) de tout niveau. La spécificité est davantage statistique, de sorte que la probabilité d'établissement d'une innervation en provenance d'un niveau donné de la moelle épinière évolue progressivement depuis les limites rostrales jusqu'aux limites caudales des ensembles cibles. Cette sélectivité de niveau est en accord avec l'existence de gradients moléculaires qui influencent mais ne 
déterminent pas strictement la formation synaptique entre des partenaires de niveaux correspondants. Notre but est d'appliquer les méthodes immunologiques du type de celles précédemment décrites pour rechercher les molécules intervenant dans cette information positionnelle.

\section{Un test biologique pour les molécules d'adhérence cellulaire de muscle énervé.}

Bien qu'aucun anticorps ou antigène discriminant les muscles rostraux des muscles caudaux n'ait encore été décrit, les travaux discutés précédemment ont permis l'identification de molécules que les muscles pouvaient utiliser pour informer leurs axones quant à leur état d'innervation et la proximité des sites synaptiques. La distribution de plusieurs de ces molécules est schématisée sur la figure 8. Leur disposition suggère que les axones en régénération rencontrent un nombre croissant d'espèces attractives lorsqu'ils vont à la rencontre des sites synaptiques primitifs. Par exemple, les muscles énervés pourraient sécréter des facteurs de croissance solubles que les axones reconnaissent à distance ; les fibres énervées d'un muscle sont riches en NCAM ; les zones périsynaptiques portent des dépôts interstitiels de plusieurs molécules d'adhérence; et la LB des sites synaptiques primitifs contient des molécules représentant l'information la plus localisée et peut-être la plus attractive.

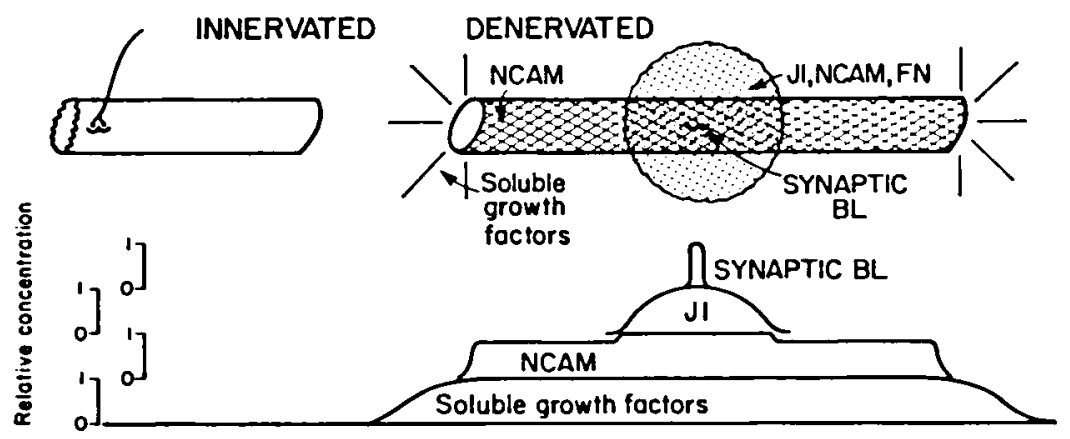

FIG. 8. - Schéma récapitulatif des molécules susceptibles d'être utilisées par les muscles énervés pour influencer la régénération axonale.

Nous aimerions utiliser les blocages immunologiques pour tester le modèle qu'impliquent ces arrangements. Malheureusement, les deux approches les plus directes semblent être mal adaptées à cet objectif. Les cocultures de neurones et de myotubes embryonnaires, qui ont été utiles pour quantité d'études sur la synaptogenèse, ne manifestent pas la distribution moléculaire topographiquement ordonnée que nous aimerions étudier. Les injections in vivo d'anticorps représentent potentiellement le test le plus direct, mais à notre avis les difficultés 
de réaliser et d'interpréter de telles expériences sur l'animal entier sont prohibitives. Nous avons donc étudié le comportement de neurones cultivés sur des sections tissulaires réalisées au cryostat, du type de celles déjà utilisées pour localiser la distribution des molécules à la surface cellulaire. Nous avons utilisé des cellules nerveuses prélevées sur le ganglion ciliaire de poulet, parce que ce sont des neurones bien caractérisés qui forment des jonctions neuromusculaires cholinergiques in vivo et in vitro, et qui sont beaucoup plus faciles à isoler que les motoneurones spinaux. Nous avons ensemencé ces neurones sur des sections de $6 \mu \mathrm{m}$ d'épaisseur, que nous avons ensuite incubés dans un milieu nutritif pendant 1 à 6 jours, puis marqués avec de l'anti-NCAM pour examiner leurs prolongements. Nos principaux résultats (Covault et al., 1987) sont les suivants :

En premier lieu, les neurones survivent et envoient des prolongements à la fois sur les sections de muscles innervés et énervés, comme sur d'autres tissus. Dans chaque cas, les neurites croissent de préférence le long des surfaces des cellules sectionnées (fig. 9a) et plusieurs résultats (détaillés dans Covault et al., 1987) montrent nettement que la croissance est guidée par des interactions spécifiques des neurites avec les molécules des surfaces cellulaires plutôt que par des hétérogénéités mécaniques dues à la section. Ainsi, le système de « cryoculture », adapté d'une méthode d'étude de l'adhérence des lymphocytes aux éléments tissulaires (Stamper and Woodruff, 1976), pourrait être intéressant pour étudier le guidage axonal sur de nombreux tissus adultes ou en développement.

Deuxièmement, les neurites sont en moyenne plus longs, plus gros et plus ramifiés sur les sections de muscles énervés que sur les sections de muscles innervés. De plus, la croissance sur les muscles énervés est plus importante sur des sections effectuées au niveau des zones riches en plaques motrices que sur des sections faites au niveau extrasynaptique. Sur une série de 16 cultures, la longueur moyenne des neurites est de $40 \pm 3 \mu \mathrm{m}$ (SE) sur des sections riches en plaque motrice de muscles innervés, et respectivement de $56 \pm 4 \mu \mathrm{m}$ et $67 \pm 4 \mu \mathrm{m}$ sur des sections de zones extrasynaptiques et synaptiques de muscles énervés. Bien que non considérables, ces différences sont cependant homogènes et significatives.

Troisièmement, les neurites qui rencontrent des nerfs intramusculaires sur la section ne poursuivent leurs croissances qu'à leur contact (fig. 9b). Les neurites croissant sur des branches ou des troncs nerveux sont considérablement plus longs que ceux croissant à la surface des cellules musculaires. Ainsi, les nerfs intramusculaires peuvent orienter la croissance axonale en cryoculture comme ils le font in vivo.

Enfin, les neurites qui atteignent les sites synaptiques primitifs s'arrêtent généralement à ce niveau (fig. 9c). Ces contacts sont rares, en raison de la faible longueur des neurites et de la rareté des sites synaptiques. Quoiqu'il en soit, ce phénomène est suffisamment net pour qu'on ne puisse douter que les axones reconnaissent certains constituants des sites synaptiques sectionnées.

Dans l'ensemble, ces résultats montrent que les axones sont susceptibles de réagir avec des molécules disposées à la surface cellulaire des muscles énervés. En outre, la cryoculture représente un modèle permettant d'effectuer des tests immunologiques de molécules que nous pensons être impliquées dans le 

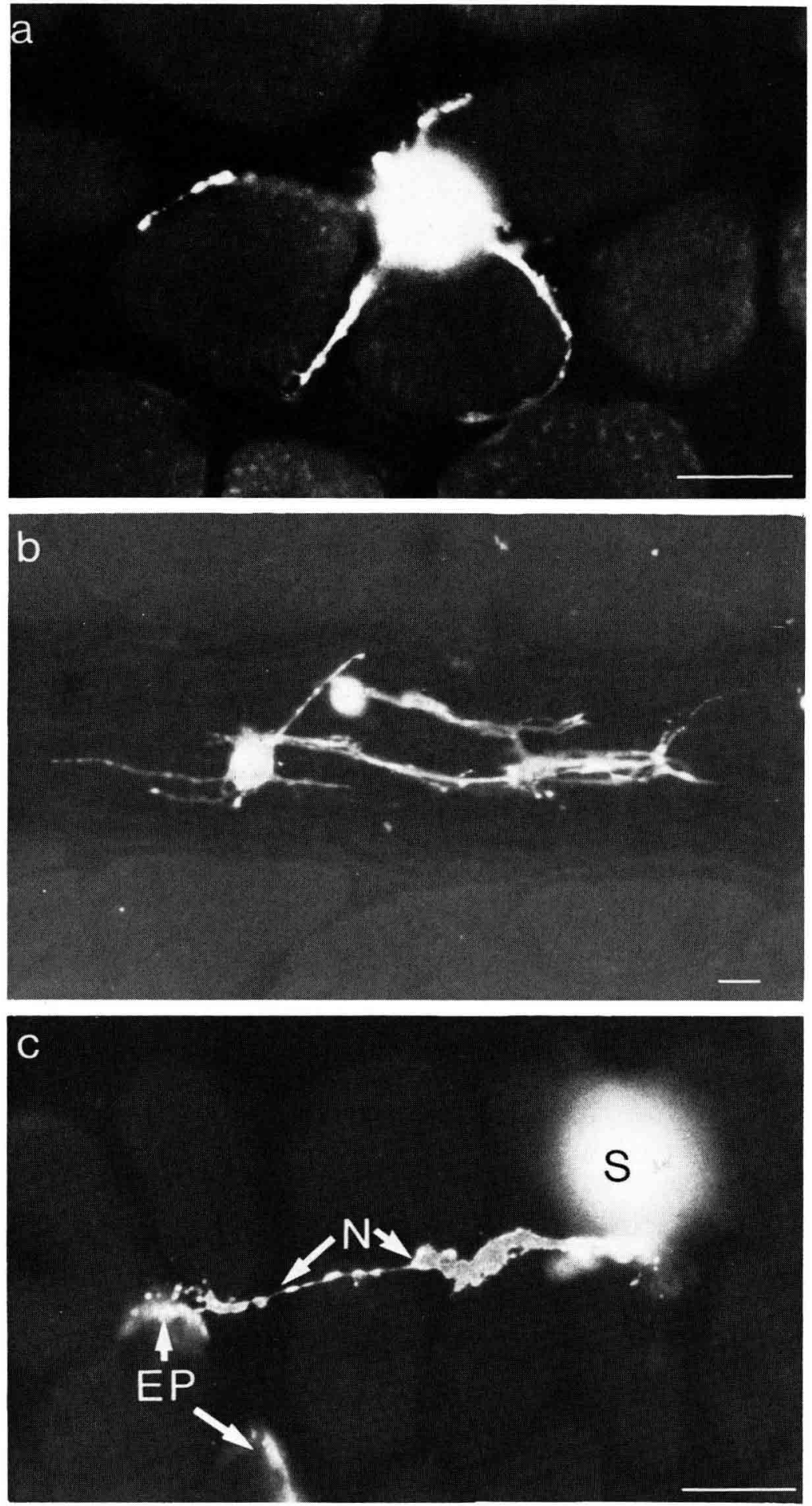

FIG. 9. - Marquage par la NCAM de neurones cultivés sur des sections au cryostat de musc/e squelettique énervé. Les neurites croissent le long de la surface des fibres musculaires en a et $c$, mais le long d'un tronc nerveux intramusculaire en $b$. Double marquage à l' $x$-bungarotoxine rhodaminée et à la NCAM en $c$, pour montrer la terminaison d'un neurite $(N)$ sur une plaque motrice (EP). Le soma neuronal (S) en $c$ est hors du champs de focalisation. Echelles $=20 \mu \mathrm{m}$. 
guidage de la réinnervation (fig. 8), modèle qui pourrait être utile dans la recherche de voies plus fines utilisées par les axones pour faire la distinction entre les muscles - c'est-à-dire, sur la base de leur position dans l'axe rostro-caudal.

Remerciements. - Traduit de l'anglais par F. Bacou.

$13^{e}$ Réunion du groupe Développement I.N.R.A., Cap d'Agde, 25-27 mai 1987

\section{Références}

AITKEN J. T., 1950. Growth of nerve implants in voluntary muscle. J. Anat., 84, 38-49.

ANDERSON M. J., FAMBROUGH D. M., 1983. Aggregates of acetylcholine receptors are associated with plaques of a basal lamina heparan sulfate proteoglycan on the surface of skeletal muscle fibers. J. Cel/ Biol., 97, 1396-1411.

ANDERSON M. J., COHEN M. W., ZORYCHTA E., 1977. Effects of innervation on the distribution of acetylcholine receptors on cultured muscle cells. J. Physiol., 268, 731-756.

BENNETT M. R., PETTIGREW A. G., 1974. The formation of synapses in reinnervated and cross-reinnervated striated muscle during development. J. Physiol., 241, 547-573.

BENNETT M. R., PETTIGREW A. G., 1976. The formation of neuromuscular synapses. Cold Spring Harbor Symp. Quant. Biol., 40, 409-424.

BERNSTEIN J. J., GUTH L., 1961. Non-selectivity in the establishment of neuromuscular connections following nerve regeneration in the rat. Exp. Neurol., 4, 262-275.

BIXBY J. L., REICHARDT L. F., 1987. Effects of antibodies to neural cell adhesion molecule $(\mathrm{N}-\mathrm{CAM})$ on the differentiation of neuromuscular contacts between ciliary ganglion neurons and myotubes in vitro. Dev. Biol., 119, 363-372.

BIXBY J. L., PRATT R. S., LILIEN J., REICHARDT L. F., 1987. Neurite outgrowth on muscle cell surfaces involves extracellular matrix receptors as well as calcium-dependent and independent cell adhesion molecules. Proc. nat. Acad. Sci. USA, 84, 2555-2558.

BOZYCZKO D., HORWITZ A. F., 1986. The participation of a putative cell surface receptor for laminin and fibronectin in peripheral neurite extension. $J$. Neurosci. 6, $1241-1251$

CASHMAN N. R., COVAULT J., WOLLMAN R. L., SANES J. R., 1987. Neural cell adhesion molecule in normal, denervated, and myopathic muscle. Ann. Neurol., 21, 481-489.

CHIQUET-EHRISMANN R., MACKIE E. J., PEARSON C. A., SAKAKURA T., 1986. Tenascin : an extracellular matrix protein involved in tissue interactions during fetal development and oncogenesis. Cell, 47, 131-139.

CHIU A. Y., MATTHEW W. D., PATTERSON P. H., 1986. A monoclonal antibody which blocks the activity of a neurite regeneration promoting factor: studies on the binding site and its localization in vivo. J. Cell Biol., 102, 1383-1398.

CONNOR E. A., MCMAHAN U. J., 1987. Cell accumulation in the junctional region of denervated muscle. J. Cell Biol., 104, 109-120.

COVAULT J., SANES J. R., 1985. Neural cell adhesion molecule (N-CAM) accumulates in denervated and paralyzed skeletal muscles. Proc. nat. Acad. Sci. USA, 82, 4544-4548.

COVAULT J., SANES J. R., 1986. Distribution of N-CAM in synaptic and extrasynaptic portions of developing and adult skeletal muscle. J. Cell Biol., 102, 716-730.

COVAULT J., CUNNINGHAM J. M., SANES J. R., 1987. Neurite outgrowth on cryostat sections of innervated and denervated skeletal muscle. J. Cell Biol., 105, 2479-2488.

COVAULT J., MERLIE J. P., GORIDIS C., SANES J. R., 1986. Molecular forms of N-CAM and its RNA in developing and denervated skeletal muscle. J. Cell Biol., 102, 731-739.

DAVIS G. E., MANTHORPE M., ENGVALL E., VARON S., 1985. Isolation and characterization of rat Schwannoma neurite-promoting factor: evidence that the factor contains laminin. $J$. Neurosci, 5, 2662-2671. 
ELIZALDE A., HEURTA M., STEFANI E., 1983. Selective reinnervation of twitch and tonic muscle fibers of the frog. J. Physiol., 340, 513-524.

ELSBERG C. A., 1917. Experiments on motor nerve regeneration and the direct neurotization of paralyzed muscles by their own and by foreign nerves. Science, 45, 318-320.

FALLON J. R., NITKIN R. M., REIST N. E., WALLACE B. G., MCMAHAN U. J., 1985. Acetylcholine receptor-aggregating factor is similar to molecules concentrated at neuromuscular junctions. Nature, 315, 571-574.

FRANK E., JANSEN J. K. S., LØMO T., WESTGAARD R. H., 1975. The interaction between foreign and original motor nerves innervating the soleus muscle of rats. J. Physiol., 247, 725-743.

GATCHALIAN C. L., CHIU A. Y., SANES J. R., 1985. Monoclonal antibodies to laminin that distinguish synaptic and extrasynaptic domains in muscle fiber basal lamina. J. Cell Biol., 101. 89a.

GATCHALIAN C. L., SANES J. R., 1987. Fibroblasts from denervated muscle synthesize NCAM, J1, and fibronectin. Soc. Neurosci. Abstr., 13, 375.

GLICKSMAN M., SANES J. R., 1983. Development of motor nerve terminals formed in the absence of muscle fibers. J. Neurocytol., 12, 661-671.

GOLDMAN D., BOULTER J., HEINEMANN S., PATRICK J., 1985. Muscle denervation increases the levels of two mRNAs coding for the acetylcholine receptor $\alpha$-subunit. J. Neurosci., 5. 2553-2558.

GRUMET M., RUTISHAUSER U., EDELMAN G. M., 1982. Neural adhesion molecule is on embryonic muscle cells and mediates adhesion to nerve cells in vitro. Nature, 295, 693-695.

GRUMET M., HOFFMAN S., CROSSIN K. L., EDELMAN G. M., 1985. Cytotactin, an extracellular matrix protein of neural and nonneural tissues that mediates glia-neuron interaction. Proc. nat. Acad. Sci. USA, 82, 8075-8079.

GURNEY M. E., APATOFF B. R., HEINRICH S. P., 1986. Suppression of terminal axonal sprouting at the neuromuscular junction by monoclonal antibodies against a muscle-derived antigen of 56,000 daltons. J. Cell Biol., 102, 2264-2272.

GUTMANN E., YOUNG J. Z., 1944. The re-innervation of muscle after various periods of atrophy. J. Anat., 78, 15-43.

HENDERSON C. E., HUCHET M., CHANGEUX J.-P., 1983. Denervation increases a neuritepromoting activity in extracts of skeletal muscle. Nature, 302, 609-611.

HUNTER D. D., SANES J. R., CHIU A. Y., 1987. An antigen concentrated in the basal lamina of the neuromuscular junction. Soc. Neurosci. Abstr., 13, 375.

HYNES R. O., 1987. Integrins : a family of cell surface receptors. Cell, 48, 549-554.

IDE C., TOHYAMA K., YOKOTA R., NITATORI T., ONODERA S., 1983. Schwann cell basal lamina and nerve regeneration. Brain Res., 288, $61-75$.

JANSEN J. K. S., LØMO T., NICOLAYSEN K., WESTGAARD R. H., 1973. Hyperinnervation of skeletal muscle fibers: dependence on muscle activity. Science, 181, 559-561.

KUROMI H., KIDIKORO Y., 1984. Nerve disperses preexisting acetylcholine receptor clusters prior to induction of receptor accumulation in Xenopus muscle cultures. Dev. Biol., 103, 53-61.

KLARSFELD A., CHANGEUX J.-P., 1985. Activity regulates the levels of acetylcholine receptor $\alpha$-subunit mRNA in cultured chicken myotubes. Proc. nat. Acad. Sci. USA, 82, 4558-4562.

KRUSE J., KEILHAUER G., FAISSNER A., TIMPL R., SCHACHNER M., 1985. The J1 glycoprotein - a novel nervous system cell adhesion molecule of the L2/HNK-1 family. Nature, 316, $146-148$.

KUFFLER D. P., 1986. Isolated satellite cells of a peripheral nerve direct the growth of regenerating frog axons. J. comp. Neurol., 249, 57-64.

LANDER A. D., FUJII D. K., REICHARDT L. F., 1985. Purification of a factor that promotes neurite outgrowth : isolation of laminin and associated molecules. J. Cell Biol., 101, 898-913.

LANDMESSER L., 1972. Pharmacological properties, cholinesterase activity and anatomy of nerve-muscle junctions in vagus-innervated frog sartorius. J. Physiol., 220, 243-256.

LASKOWSKI M. B., SANES J. R., 1987a. Topographic mapping of motor pools onto skeletal muscles. J. Neurosci, 7, 252-260.

LASKOWSKI M. B., SANES J. R., 1987b. Topographically selective reinnervation of adult mammalian muscles. Soc. Neurosci. Abstr., 13, 1422. 
LETINSKY M. K., FISCHBACH G. D., McMAHAN U. J., 1976. Precision of reinnervation of original postsynaptic sites in muscle after a nerve crush. J. Neurocytol., 5, 691-718.

MERLIE J.P., SANES J.R., 1985. Concentration of acetylcholine receptor mRNA in synaptic regions of adult muscle fibres. Nature, 317, 66-68.

MERLIE J. P., SANES J. R., 1986. Regulation of synapse-specific genes. In : R. LEVI-MONTALCINI et a/. eds. Molecular aspects of neurobiology. Berlin Springer-Verlag.

MERLIE J. P., ISENBERG K. E., RUSSELL S. D., SANES J. R., 1984. Denervation supersensitivity in skeletal muscle: analysis with a cloned cDNA probe. J. Cell Biol., 99, 332-335.

MOORE S. E., WALSH F. S., 1986. Nerve-dependent regulation of neural cell adhesion molecule expression in skeletal muscle. Neuroscience, 18, 499-505.

MURRAY M. A., ROBBINS N., 1982. Cell proliferation in denervated muscle : identity and origin of the dividing cells. Neuroscience, 7, 1823-1833.

NURCOMBE V., HILL M. A., EAGELSON K. L., BENNETT M. R., 1984. Motor neuron survival and neuritic extension from spinal cord explants induced by factors released from skeletal muscle. Brain Res., 291, 19-28.

PURVES D., THOMPSON W., YIP J. W., 1981. Reinnervation of ganglia transplanted to the neck from different levels of the guinea-pig sympathetic chain. J. Physiol., 313, 49-63.

RAMON Y CAJAL S., 1928, reprinted 1968. Degeneration and regeneration of the nervous system. Hafner Publishing, London.

RIEGER F., GRUMET M., EDELMAN G. M., 1985. N-CAM at the vertebrate neuromuscular junction. J. Cell Biol., 101, 285-293.

RUTISHAUSER U., GRUMET M., EDELMAN G. M., 1983. Neural cell adhesion molecule mediates initial interactions between spinal cord neurons and muscle cells in culture. J. Cel/ Biol., 97. $145-152$.

SALPETER M. M., LORING R. H., 1985. Nicotinic acetylcholine receptors in vertebrate muscle: properties, distribution and neural control. Progr. Neurobiol., 25, 297-325.

SANES J. R., 1982. Laminin, fibronectin and collagen in synaptic and extrasynaptic portions of muscle fiber basement membrane. J. Cell Biol., 93, 442-451.

SANES J. R., HALL Z. W., 1979. Antibodies that bind specifically to synaptic sites on muscle fiber basal lamina. J. Cell Biol., 83, 357-370.

SANES J. R., CHENEY J. M., 1982. Lectin-binding reveals a synapse-specific carbohydrate in skeletal muscle. Nature, 300, 646-647.

SANES J. R., CHIU A. Y., 1983. The basal lamina of the neuromuscular junction. Cold Spring Harbor Symp. Quant. Biol., 48, 667-678.

SANES J. R., COVAULT J., 1985. Axon guidance during reinnervation of skeletal muscle. Trends Neurosci, 8, 523-528.

SANES J. R., MARSHALL L. M., McMAHAN U. J., 1978. Reinnervation of muscle fiber basal lamina after removal of myofibers. Differentiation of regenerating axons at original synaptic sites. J. Cel/ Biol., 78, 176-198.

SANES J. R., SCHACHNER M., COVAULT J., 1986. Expression of several adhesive macromolecules (N-CAM, L1, J1, NILE, uvomorulin, laminin, fibronectin and a heparan sulfate proteoglycan) in embryonic, adult and denervated adult skeletal muscles. J. Ce/l Biol., 102, 420-431.

SCHERER S. S., EASTER S. S., 1984. Degenerative and regenerative changes in the trochlear nerve of goldfish. J. Neurocytol., 13, 519-565.

SCOTT L. J., SANES J. R., 1984. A lectin that selectively stains neuromuscular junctions binds to collagen-tailed acetylcholinesterase. Soc. Neurosci. Abstr., 10, 546.

SCOTT L. J., BACOU F., SANES J. R., 1988. A synapse-specific carbohydrate at the neuromuscular junction: association with both acetylcholinesterase and a glycolipid. J. Neurosci., 8 , 932-944.

SLACK J. R., HOPKINS W. G., POCKETT S., 1983. Evidence for a motor nerve growth factor. Muscle Nerve, 6, 243-252.

STAMPER H. B., WOODRUFF J. J., 1976. Lymphocyte homing into lymph nodes: in vitro demonstration of the selective affinity of recirculating lymphocytes for high-endothelial venules. J. exp. Med., 144, 828-833. 
TELLO F., 1907. Dégénération et régénération des plaques motrices après la section des nerfs. Trav. Lab. Rech. Biol., 5, 117-149.

WEINBERG C. B., SANES J. R., HALL Z. W., 1981. Formation of neuromuscular junctions in adult rats: accumulation of acetylcholine receptors, acetylcholinesterase, and components of synaptic basal lamina. Dev. Biol., 84, 255-266.

WIGSTON D. J., 1986. Selective innervation of transplanted limb muscles by regenerating motor axons in the axolotl. J. Neurosci, 6, 2757-2763.

WIGSTON D. J., SANES J. R., 1982. Selective reinnervation of adult mammalian muscles by axons from different segmental levels. Nature, 299, 464-467.

WIGSTON D. J., SANES J. R., 1985. Selective reinnervation of rat intercostal muscles transplanted from different segmental levels to a common site. J. Neurosci., 5, 1208-1221. 